\title{
Defining when to offer operative (Q) crossarar treatment for intrahepatic cholangiocarcinoma: A regret-based decision curves analysis
}

\author{
Fabio Bagante, MD, ${ }^{a}$ Gaya Spolverato, MD, ${ }^{a}$ Alessandro Cucchetti, MD, ${ }^{\mathbf{b}}$ Faiz Gani, MBBS, ${ }^{\text {a }}$ \\ Irinel Popescu, MD, ${ }^{\mathrm{c}}$ Andrea Ruzzenente, MD, ${ }^{\mathrm{d}}$ Hugo P. Marques, MD, ${ }^{\mathrm{e}}$ Luca Aldrighetti, MD, ${ }^{\mathrm{f}}$ \\ T. Clark Gamblin, MD, ${ }^{\mathrm{g}}$ Shishir K. Maithel, MD, ${ }^{\mathrm{h}}$ Charbel Sandroussi, MD, ${ }^{\mathrm{i}}$ Todd W. Bauer, MD, ${ }^{\mathrm{j}}$ \\ Feng Shen, MD, ${ }^{\mathrm{k}}$ George A. Poultsides, MD, ${ }^{1}$ James Wallis Marsh, $\mathrm{MD}^{\mathrm{m}}{ }^{\mathrm{A}}$ Alfredo Guglielmi, $\mathrm{MD},{ }^{\mathrm{d}}$ and \\ Timothy M. Pawlik, MD, MPH, PhD, FACS, ${ }^{\mathbf{a}}$ Baltimore, MD, Bologna, Verona, Milan, Italy, Bucharest, \\ Romania, Lisbon, Portugal, Milwaukee, WI, Atlanta, GA, Sydney, Australia, Charlottesville, VA, Shanghai, \\ China, Stanford, CA, and Pittsburgh, PA
}

\begin{abstract}
Background. Regret-based decision curve analysis (DCA) is a framework that assesses the medical decision process according to physician attitudes (expected regret) relative to disease-based factors. We sought to apply this methodology to decisions around the operative management of intrahepatic cholangiocarcinoma (ICC).

Methods. Utilizing a multicentric database of 799 patients who underwent liver resection for ICC, we developed a prognostic nomogram. DCA tested 3 strategies: (1) perform an operation on all patients, (2) never perform an operation, and (3) use the nomogram to select patients for an operation.

Results. Four preoperative variables were included in the nomogram: major vascular invasion $(H R=1.36)$, tumor number (multifocal, $H R=1.18)$, tumor size $(>5 \mathrm{~cm}, H R=1.45)$, and suspicious lymph nodes on imaging $(H R=1.47$; all $\mathrm{P}<.05)$. The regret-DCA was assessed using an online survey of 50 physicians, expert in the treatment of ICC. For a patient with a multifocal ICC, largest lesion measuring $>5 \mathrm{~cm}$, one suspicious malignant lymph node, and vascular invasion on imaging, the 1-year predicted survival was $52 \%$ according to the nomogram. Based on the therapeutic decision of the regret-DCA, $60 \%$ of physicians would advise against an operation for this scenario. Conversely, all physicians recommended an operation to a patient with an early ICC (single nodule measuring $3 \mathrm{~cm}, n o$ suspicious lymph nodes, and no vascular invasion at imaging).

Conclusion. By integrating a nomogram based on preoperative variables and a regret-based DCA, we were able to define the elements of how decisions rely on medical knowledge (postoperative survival predicted by a nomogram, severity disease assessment) and physician attitudes (regret of commission and omission). (Surgery 2016;160:106-17.)
\end{abstract}

From the Johns Hopkins University School of Medicine, ${ }^{a}$ Baltimore, MD; University of Bologna, Ospedale S. Orsola-Malpighi, ${ }^{b}$ Bologna, Italy; Fundeni Clinical Institute, ${ }^{c}$ Bucharest, Romania; University of Verona, Policlinico GB Rossi, ${ }^{d}$ Verona, Italy; Curry Cabral Hospital, ${ }^{e}$ Lisbon, Portugal; Ospedale San Raffaele, ${ }^{f}$ Milan, Italy; Medical College of Wisconsin, ${ }^{g}$ Milwaukee, WI; Emory University, ${ }^{h}$ Atlanta, GA; University of Sydney, ${ }^{i}$ Sydney, Australia; University of Virginia, ${ }^{j}$ Charlottesville, VA; Eastern Hepatobiliary Surgery Hospital, ${ }^{k}$ Shanghai, China; Stanford University, ${ }^{l}$ Stanford, CA; and University of Pittsburgh Medical Center ${ }^{m}$, Pittsburgh, PA

Accepted for publication January 30, 2016.

Reprint requests: Timothy M. Pawlik, MD, MPH, PhD, FACS, Division of Surgical Oncology, Department of Surgery, Johns Hopkins Hospital, Baltimore, MD 21287. E-mail: tpawlik1@ jhmi.edu.

0039-6060/\$ - see front matter

(c) 2016 Elsevier Inc. All rights reserved.

http:/ /dx.doi.org/10.1016/j.surg.2016.01.023
INTRAHEPATIC CHOLANGIOCARCINOMA (ICC) accounts for $10-15 \%$ of all liver cancers and after hepatocellular carcinoma, is the second most common primary liver malignancy. ${ }^{1,2}$ An operation remains the sole curative treatment for ICC; however, only $30-40 \%$ of patients present with resectable disease at the time of diagnosis. ${ }^{3}$ High recurrence rates and inefficient adjuvant chemotherapy regimens 
have contributed to a poor 5-year survival ranging from $14-40 \% .{ }^{4,5}$ In fact, a multicentric international study of patients with ICC reported that up to $25 \%$ of patients presented with resectable disease and had a median postoperative overall survival of only 14.8 months. ${ }^{6}$

These survival data are not too dissimilar to a recent phase 3, randomized control trial of patients with advanced unresectable ICC, who achieved a median survival of 11.7 months with the use of combination gemcitabine and cisplatin chemotherapy. ${ }^{7}$ As such, it appears that a subset of patients may derive little to no additional survival benefit from liver resection compared with chemotherapy alone, while at the same time being exposed to the risk of postoperative morbidity. ${ }^{8,9}$ Data on the cost-effectiveness of upfront hepatic resection versus initial systemic chemotherapy followed by possible curative hepatic resection further underline the need for more precise guidelines on the operative management of patients with advanced ICG. ${ }^{10}$

Given the complexity and challenges involved in decision-making around the management of patients with ICC, traditional decision-making utility models may be inadequate. In utility theory, the axiom of decision analysis requires that the physician make decisions based on maximizing the expected utility outcome (eg, prolongation of patient survival) but does not allow for any estimation of the uncertainty in achieving that outcome. In reality, however, a physician's choice of therapy may depend greatly on the amount of uncertainty surrounding the likelihood of achieving the outcome. ${ }^{11}$ For example, after making a decision that involves some uncertainty, the physician may discover, when learning the relevant outcomes, that an alternative approach would have been preferred-thereby bringing about a sense of regret. In particular, a physician might have preferred to avoid certain consequences (eg, postoperative complications) in which he/she comes to realize that the desired outcome (eg, longterm survival) was not achieved. ${ }^{12}$

In contrast to utility-based theory, regret-based decision methodology is a framework that integrates medical knowledge and physician attitudes (expected regret) when outcomes have a level of uncertainty. ${ }^{13,14}$ In regret theory, the decision maker relies on a combination of rationality and intuition, expecting that some outcomes will be associated with high regret, which he / she would like to avoid. ${ }^{12,13,15-17}$ In this model of decisionmaking, the optimal choice would be the one associated with the least amount of regret.
In the current study, we sought to apply regretbased decision curve analysis (regret-DCA) to different treatment strategies for patients presenting with ICC. Specifically, we assessed if decisions regarding whether to perform an operation or treat with chemotherapy alone can be aided by a prognostic model derived from a large, multicenter, international cohort of patients with ICC, depending on regret-based thresholds. Furthermore, we sought to compare the actual attitudes of providers using a case-based survey versus the results of the regret-DCA model.

\section{MATERIALS AND METHODS}

Data sources and study population. Data on patients with histologically confirmed ICC were obtained from an international, multi-institutional database of 799 patients who underwent a liver operation between 1990 and 2013 at 14 major hepatobiliary centers (Johns Hopkins Hospital, Baltimore, MD; Medical College of Wisconsin, Milwaukee, WI; Stanford University, Stanford, CA; University of Virginia, Charlottesville, VA; Emory University, Atlanta, GA; University of Pittsburgh, Pittsburgh, PA; Fundeni Clinical Institute of Digestive Disease, Bucharest, Romania; Curry Cabral Hospital, Lisbon, Portugal; Hopitaux Universitaires De Geneve, Geneva, Switzerland; Ospedale San Raffaele, Milan, Italy; University of Sydney, Sydney, Australia; Eastern Hepatobiliary Surgery Hospital, Shanghai, China; Ospedale GB Rossi, University of Verona, Italy; Ospedale S. Orsola, University of Bologna, Italy).

Patients presenting with metastatic disease at the time of diagnosis (American Joint Committee on Cancer Stage IVB) were excluded from the study cohort. Similarly, patients who underwent a palliative resection or patients treated with percutaneous ablation were excluded from the study population. Data were collected on a wide range of demographic, clinical, and therapeutic factors as described earlier. ${ }^{6}$

Statistical analysis and development of the predictive model. Survival curves were estimated using the Kaplan-Meier method, and differences between curves were investigated using the logrank test. A new model to predict overall survival after an operation was created by analyzing patient and tumor characteristics. Preoperative variables that were statistically significant in the univariable analysis were tested in multivariable analyses using a Cox proportional hazards regression model. Using a backward elimination method based on the Akaike Information Criterion (AIC), variables 
were assessed for inclusion into the multivariable model. A $P$ value of .10 was the threshold for inclusion of variables in the multivariable model.

Point estimates for variables obtained from the multivariable model were reported as hazard ratios (HR) with corresponding 95\% confidence intervals $(\mathrm{CI})$.

A nomogram to predict survival of patients with ICC undergoing operative resection was built using the results of the multivariable analysis. Specifically, the preoperative variables that were statistically significant in both the univariate and multivariable analyses were included in the nomogram. The nomogram was developed using the package of Hmisc in R version 3.11.0 (http:/ / www. r-project.org/). The concordance index (C-index) was used to assess the performance of the nomogram, while the calibration of the nomogram was evaluated by comparing nomogram-predicted versus observed Kaplan-Meier estimates of survival probability. Bootstrap with 5,000 resample was used for these analyses.

Regret-based decision analysis. In the regretDCA, the decision was analyzed comparing the probability of an outcome (eg, probability of survival $[\mathrm{S}]$ ) with a regret threshold probability (Preg) based on the physician attitudes. The $\mathrm{S}$ was estimated using the nomogram including relevant preoperative clinicopathologic variables. Preg was estimated using Equation 1:

Equation 1 for Preg : $\frac{1}{1+\frac{\text { Regret of Omission }}{\text { Regret of Commision }}}$

Regret of omission was defined as the regret felt by the physician who decided not to perform an operation on a patient with ICC, even if he/she might have benefited from the treatment. Conversely, regret of commission was defined as the regret felt by the physician who decided to perform an operation on a patient, even if he/she might not have gained any benefit from the treatment. $^{13-15}$

The results of the DCA analyses were assessed using the probability of survival at different time points $(1,2$, and 3 years). In other words, we considered the hypothesis that different physicians might have different opinions on what survival time point would be relevant in deciding whether to offer a patient resection for ICC. Consequently, we evaluated the overall $\mathrm{S}$ calculated at 1,2 , and 3 years in relation to the Preg expressed by the physician. In the DCA model, an operation should be offered to patients if the patient's $S$ was equal to or above the Preg ( $\mathrm{S} \geq$ Preg), because this decision would be associated with the least amount of regret.

Regret associated with the treatment choice was analyzed using 3 different strategies: (1) a physician could perform operations on all patients with technically resectable ICC; (2) a physician could decide to treat all patients with chemotherapy and advise against performing an operation in any patient; or (3) a physician could use the prediction model (nomogram) to select patients for an operation. Any of these strategies may result in regret if the patient's survival is less than expected by the physician, while the optimal strategy would be the one associated with the least amount of regret when proven to be wrong ( $\mathrm{S}<$ Preg).

Using the decision tree reported by Djulbegovic et $\mathrm{al}^{11,12}$ (Fig 1), regret was expressed as the difference between the utility of the outcome of the treatment selected and the utility of the outcome of the action that, retrospectively, should have been taken:

Equation 2, Expected regret using the model :

$$
\frac{F P}{n} \times \frac{\text { Preg }}{1-\text { Preg }}+\frac{F N}{n}
$$

Equation 3, Expected regret for performing an

$$
\text { operation on all patients : }(1-\mathrm{S}) \times\left(\frac{\text { Preg }}{1-\text { Preg }}\right)
$$

Equation 4, Expected regret for performing no

$$
\text { operation on all patients : } \mathrm{S}
$$

where false positive was the number of patients who died within a specific temporal endpoint $(1,2$, and 3 years) and for whom $\mathrm{S}>$ Preg; false negative was the number of patients who will survive longer than a specific temporal end point (1, 2, and 3 years) and for whom $\mathrm{S}<$ Preg; $n$ was the number of patients and $\mathrm{S}$ was the overall survival probability at a specific temporal end point. All analyses were performed using STATA version 12.0 (StataCorp LP, College Station, TX) or R software for statistical computing (R Foundation, Vienna, Austria), v. 3.0.2 34, with the additional packages: survival and Hmisc. ${ }^{18-20}$

Description of the survey. To further explore the regret-DCA, an online survey was administered to examine the regret associated with treatment decisions for the 3 hypothetical ICG cases. The survey was administered to a convenience sample of 50 physicians with expertise in the treatment of ICC. Respondents were asked to report an estimation of their regret of commission and omission on 


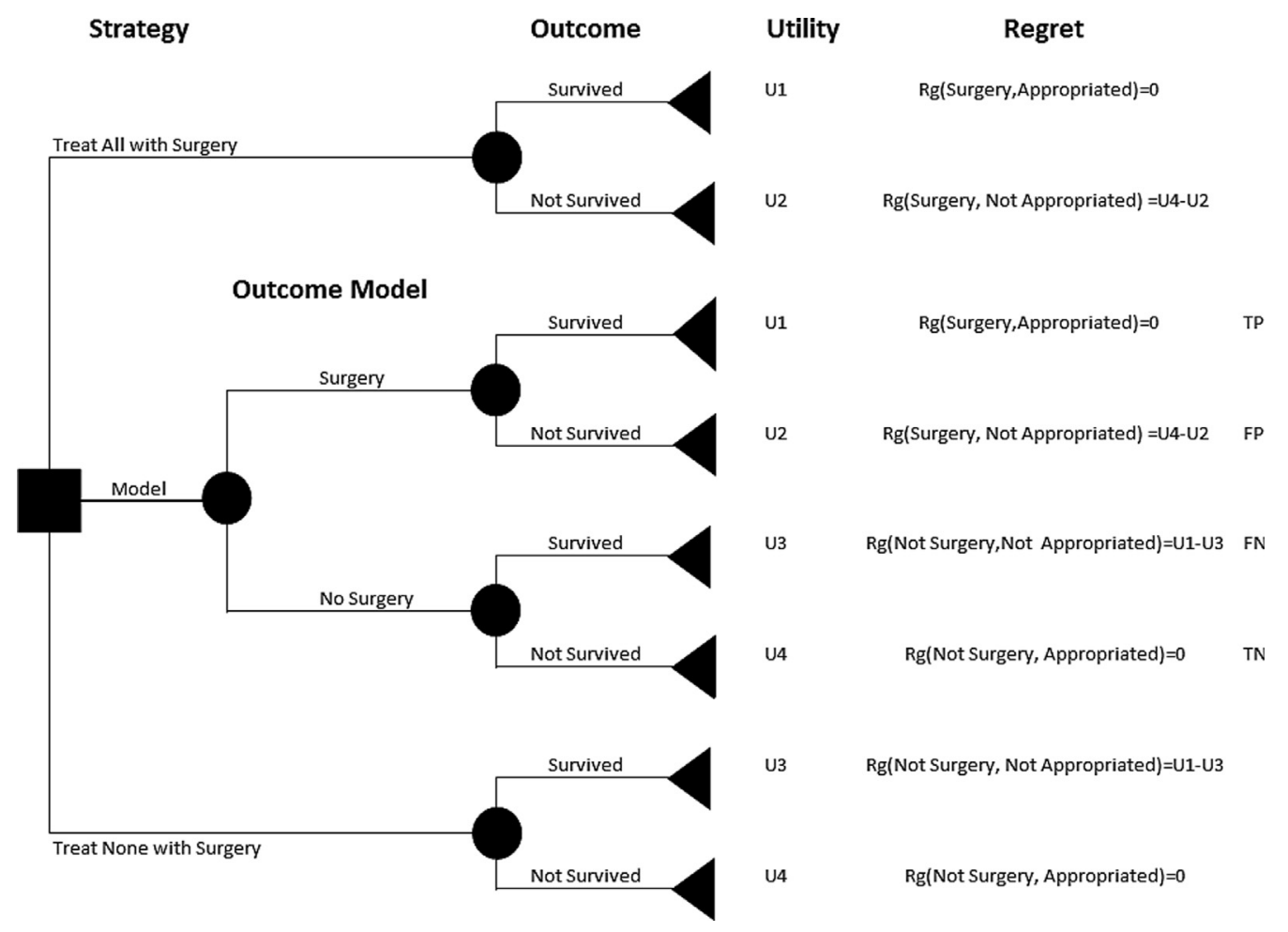

Fig 1. The decision tree for recommending an operation to ICC patients.

a scale ranging from 0 to 100 . The base-case consisted of a 65-year-old man, American Society of Anesthesiologists score II with well-controlled hypertension, normal hepatic function, and no history of chronic liver disease. The computed tomography (CT) scan performed for abdominal pain showed a nonmetastatic, operatively resectable ICC. For the purpose of each case, the physician assumed the patient was willing to proceed with the therapy recommended and had no preference for operative or nonoperative therapy.

Three different cases were described: Case 1: CT demonstrated one nodule measuring $5 \mathrm{~cm}$ located in liver segment 8 , one possible metastatic perihilar lymph node, no invasion of major vessels. Case 2: CT demonstrated one nodule measuring $3 \mathrm{~cm}$ located in liver segment 3 , no suspicious lymph nodes, no invasion of major vessels. Case 3: CT scan demonstrated 3 nodules, the largest measuring $6 \mathrm{~cm}$, located in liver segments 5 and 8 , one perihilar lymph node highly suspicious for metastasis, and vascular invasion of the right branch of the portal vein. Moreover, for each case, the physician was asked to answer the following question: "When you suggest surgery to this patient, what do you think would be an acceptable minimal survival for him/her after surgery (ie, a survival time after which you would not experience regret for having decided to operate)?”

\section{RESULTS}

Baseline characteristics. The clinicopathologic characteristics of 799 patients who underwent liver resection for ICC are presented in Table I. The majority of patients were male $(n=425 ; 53.2 \%)$, and the median age at diagnosis was 61.7 years (interquartile range [IQR] 52.0-70.1). On preoperative assessment, median CA 19-9 was $87.8 \mathrm{U} / \mathrm{mL}$ (IQR, 16.9-500.0); 188 (23.5\%) patients had lymph nodes suspicious for metastasis (ie, $>1 \mathrm{~cm}$, enhancing, etc); and 142 (17.8\%) patients had major vascular invasion on imaging (Table I); 79 (9.9\%) patients had underlying chronic liver disease.

A total of $315(39.4 \%)$ patients had multifocal disease; median tumor size was $6.5 \mathrm{~cm}$ (IQR, 4.6$9.0)$, and $516(64.6 \%)$ patients had a tumor $>5 \mathrm{~cm}$. At the time of the operation, hepatic resection consisted of a minor ( $\leq 3$ liver segments, $n=277$; $34.7 \%$ ) or a major ( $>3$ liver segments, $n=522$; $65.3 \%$ ) hepatectomy. On the final pathologic report, most patients had a well-differentiated tumor $(n=574,71.8 \%)$, while fewer had a moderately/poorly differentiated tumor $(n=225$, 
Table I. Baseline characteristics of the 799 patients who underwent liver resection for intrahepatic cholangiocarcinoma between 1990 and 2013

\begin{tabular}{lc}
\hline & $\mathrm{N}(\%)$ \\
\hline Age (y), median (IQR) & $61.7(52.0-70.1)$ \\
Gender, male & $425(53.2)$ \\
Cirrhosis, present & $79(9.9)$ \\
Margin Status, positive & $167(20.9)$ \\
Number of Tumors, multifocal & $315(39.4)$ \\
Tumor Size (cm), median (IQR) & $6.5(4.6-9.0)$ \\
Tumor Size, $\geq 5$ cm & $516(64.6)$ \\
Tumor Grade, moderate/poor & $225(28.2)$ \\
Suspicious Nodes on Preop & $188(23.5)$ \\
$\quad$ Imaging, present & $142(17.8)$ \\
Major Vascular Invasion, & \\
$\quad$ present & $301(37.7)$ \\
Microscopic Vascular Invasion, & \\
$\quad$ present & $228(28.5)$ \\
Perineural Invasion & $113(14.1)$ \\
Invasion of Adjacent Organs, & \\
$\quad$ present & $87.8(16.9-500.0)$ \\
CA19-9 (U/mL), median (IQR) & $522(65.3)$ \\
Type of Resection, major & $424(53.1)$ \\
Adjuvant Chemotherapy, & \\
$\quad$ performed & $511(63.9)$ \\
Patient Status, death & $56.0(52.5-59.8)$ \\
\hline Overall & $30.6(27.0-34.6)$ \\
Survival & $25.2(21.7-29.2)$ \\
\hline 1 y & \\
2 y y & \\
4 y & \\
5 y & \\
\hline
\end{tabular}

$C I$, Confidence interval; IQR, interquartile range; Preop, preoperative.

28.2\%). Microscopic vascular invasion $(n=301$, $37.7 \%)$ and perineural invasion $(n=228,28.5 \%)$ were also noted in a subset of patients.

Survival analysis and development of the prognostic model. With a median follow-up of 20.1 months (IQR, 10.6-38.7), 511 (63.9\%) patients died. The 1-, 2-, and 3-year survival was $77.6 \%$ (95\% CI, 74.7-80.6), 56.0\% (95\% CI, 52.559.8 ), and $41.4 \%$ (95\% CI, 37.7-45.3), respectively.

Demographic and tumor characteristics were examined for their association with survival following resection of ICC (Table II). Performing a stepwise model selection by AIC (backward selection) for the Cox proportional hazards regression model, 4 preoperative variables were found to be strongly associated with overall survival following resection: lymph nodes suspicious for metastatic disease $(\mathrm{HR}=1.47 ; 95 \% \mathrm{CI}, 1.19-1.81 ; P<.001)$, tumor size $(>5 \mathrm{~cm} ; \mathrm{HR}=1.45 ; 95 \% \mathrm{CI}, 1.19$ $1.75 ; \quad P<.001)$, major vascular invasion
$(\mathrm{HR}=1.36 ; 95 \% \mathrm{CI}, 1.08-1.73 ; P=.010)$, and number of tumors (multifocal disease, $\mathrm{HR}=1.18 ; 95 \%$ CI, 0.98-1.42; $P=.072$ ).

Using these preoperative factors, a nomogram was constructed to predict postoperative overall survival following liver resection of ICC (Fig 2). Based on the different beta coefficients, each factor was assigned a weighted number of points in the nomogram. The sum of points for each patient was associated with a specific predicted 1-, 2- and 3-year overall survival, with a higher score being associated with a worse prognosis. Discrimination ability of the final model for overall survival was assessed using the c-statistic, with a bootstrapping resample method $(n=5,000)$ yielding an unbiased c-statistic estimate of 0.59 . The calibration plots revealed good prediction of 1-, 2-, and 3-year overall survival (Supplementary Figs $A, B$, and $C$ ). The predicted probabilities overlapped with the actual survival probabilities, suggesting that the model had a good discrimination ability to distinguish high-risk from low-risk individuals.

Regret-DCA. Regret-DCA was applied to the 1-, 2-, and 3-year survival predictions. Figure 3, A, B, and $C$ depicts how the expected regret changed for different Pregs applied to 1-, 2-, and 3-year survival predictions. Specifically, these graphs analyzed the relationship between the Preg and the expected regret associated with each of the 3 possible therapeutic strategies (ie, treat all patients with an operation, treat none with an operation, and use the nomogram to select patients for an operation).

As depicted in Fig 3, A, for a Preg $<75 \%$, the least regretful strategy was to use the predictive nomogram model to identify which patients to offer an operation. In this scenario, a physician should offer liver resection only to patients with a predictive survival (estimated by the nomogram) that was greater than the Preg accepted by the physician $(\mathrm{S}>\mathrm{Preg})$. If the estimated survival based on the model's prediction was lower than the regret probability $(\mathrm{S}<\mathrm{Preg})$, then avoiding an operation and treating with chemotherapy alone would be the less regretful, and therefore preferred, strategy.

As such, for a Preg $>75 \%$, the preferred, leastregretful therapeutic strategy would be to withhold resection and offer chemotherapy alone to all patients. To demonstrate this point, take for example, a physician who may calculate his/her Preg to be $33.3 \%$ (regret of omission $=60$; regret of commission $=30$ ). If on preoperative imaging the patient had a single $6-\mathrm{cm}$ tumor with no major invasion but suspicious lymph node disease, the 
Table II. Uni- and multivariable survival analysis of the clinicopathologic characteristics of patients who underwent liver resection for intrahepatic cholangiocarcinoma

\begin{tabular}{|c|c|c|c|c|c|c|}
\hline & \multicolumn{3}{|c|}{ Univariable analysis } & \multicolumn{3}{|c|}{ Multivariable analysis } \\
\hline & $H R$ & $95 \% C I$ & $\mathrm{P} *$ & $H R$ & $95 \% C I$ & $\mathrm{P}_{\dagger}^{\dagger}$ \\
\hline Age, y & 1.01 & $0.99-1.01$ & $.099 \ddagger$ & & & \\
\hline Gender, female & 0.81 & $0.68-0.96$ & $.018 \ddagger$ & & & \\
\hline Cirrhosis, present & 1.25 & $0.94-1.65$ & .127 & & & \\
\hline Major Vascular Invasion, present & 1.56 & $1.24-1.96$ & $<.001 \ddagger$ & 1.36 & $1.08-1.73$ & .010 \\
\hline Microscopic Vascular Invasion, present & 1.21 & $1.01-1.45$ & .037 & & & \\
\hline Invasion of Adjacent Organs, present & 1.55 & $1.22-1.97$ & $<.001 \ddagger$ & & & \\
\hline Perineural Invasion, present & 1.13 & $0.93-1.38$ & .216 & & & \\
\hline Number of Tumors, multiple & 1.31 & $1.10-1.56$ & $.002 \ddagger$ & 1.18 & $0.98-1.42$ & 072 \\
\hline Margins, positive & 1.43 & $1.14-1.79$ & .001 & & & \\
\hline CA $19-9,>40 \mathrm{U} / \mathrm{mL}$ & 1.21 & $1.01-1.46$ & $.041 \ddagger$ & & & \\
\hline Tumor Size, $\geq 5 \mathrm{~cm}$ & 1.46 & $1.21-1.76$ & $<.001 \ddagger$ & 1.45 & $1.19-1.75$ & $<.001$ \\
\hline Tumor Grade, moderate/poor & 1.31 & $1.09-1.58$ & .004 & & & \\
\hline Major Resection, performed & 1.26 & $1.05-1.52$ & .012 & & & \\
\hline Suspicious Nodes on Preop Imaging, present & 1.62 & $1.33-1.98$ & $<.001 \ddagger$ & 1.47 & $1.19-1.81$ & $<.001$ \\
\hline Adjuvant Chemotherapy, performed & 0.74 & $0.62-0.88$ & $<.001$ & & & \\
\hline
\end{tabular}

* $P$ of the log-rank test comparing two survival curves.

$\dagger P$ of the $\chi^{2}$ test comparing the variables in the Cox multivariable model.

$\ddagger$ Preoperative variables tested in the multivariable model.

$H R$, Hazard ratio; CI, confidence interval; Preop, preoperative.

Points

Number of Nodules

Tumor Size

Node Status

Major Vascular Invasion

Total Points

Survival at 1 year

Survival at 2 years

Survival at 3 years

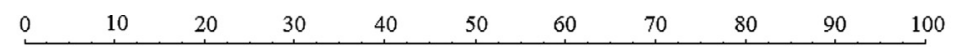

Multifocal

$$
\text { Singular }
$$
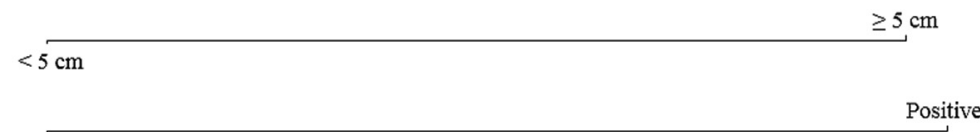

Negative

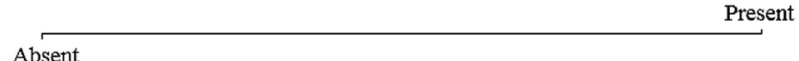

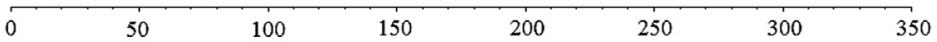

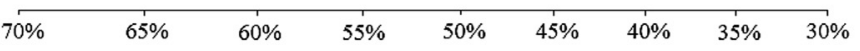

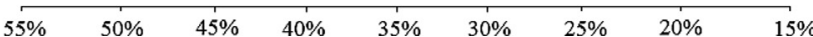

Fig 2. A nomogram, based on 4 preoperative variables, able to predict survival at 1 , 2, and 3 years. Each variable corresponds to a point (first line), and the sum of the partial points (total points) determines the predictive survival probability.

patient's predicted 1-year survival based on the nomogram model would be $66.0 \%$. In this situation, an operation should be recommended because the predicted 1-year survival was higher than the physician threshold of regret $(S>$ Preg $)$, and therefore, an operation is the least regretful treatment strategy.
However, for a Preg $>75 \%$, an operation should not be recommended regardless of the model's prediction, because the regret associated with the decision to "avoid to perform surgery to any patients" becomes equal to the regret of making the choice based on the model. In addition, given that the highest predicted 1-year 


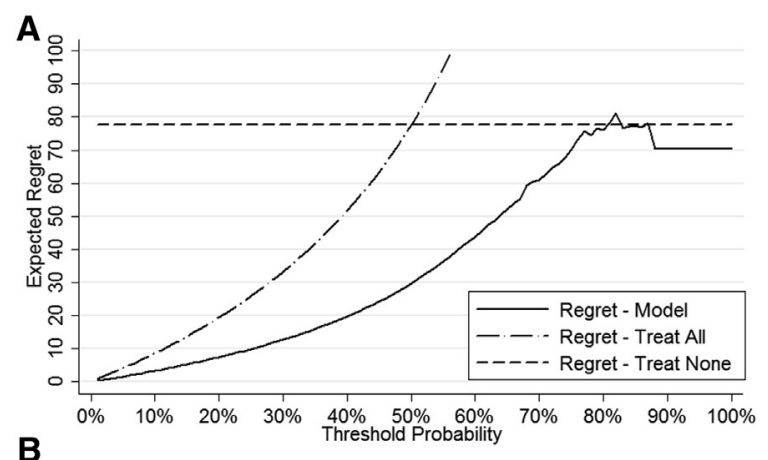

B
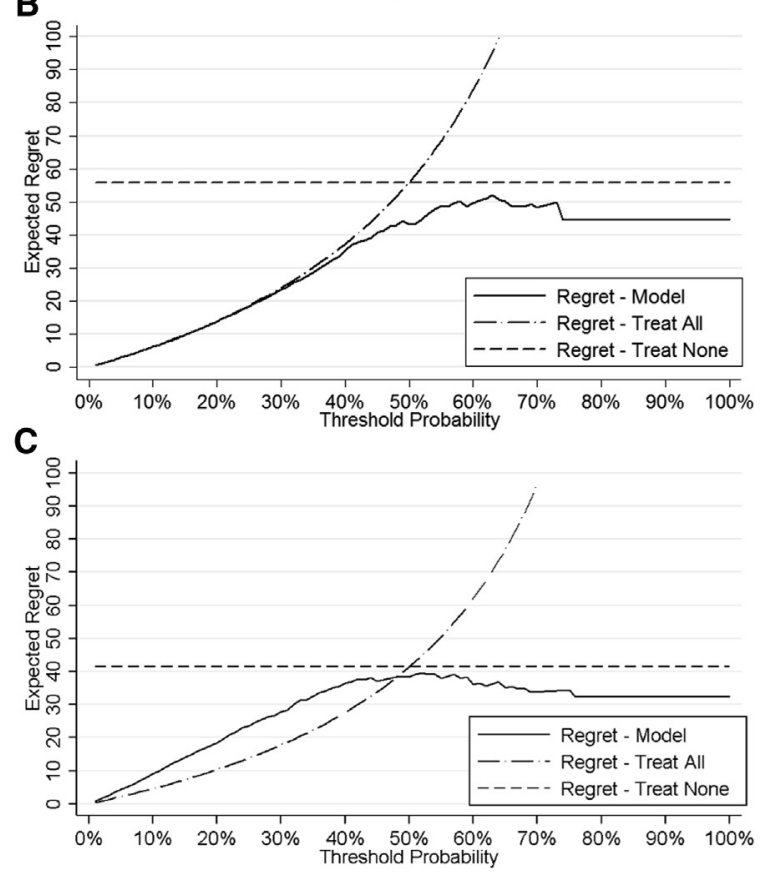

Fig 3. Regret-based decision curves analysis at $(A) 1$ year, $(B) 2$ years, and $(C) 3$ years after liver resection.

survival of the model was $87 \%$, the model would not be able to support the physician decisionmaking process for a Preg above this value.

Figure 3, $B$ and $C$ illustrates how the relationship between expected regret changes for different Pregs associated with 2- and 3-year overall survival. If a physician is considering 2-year survival as the outcome, regret-DCA would suggest that the least regretful strategy would be to use the predictive model to recommend patients for an operation when the Preg is $>30 \%$. For values $<30 \%$, both "treat all patients with surgery" and "use a predictive model to select patients who would benefit from surgery" resulted in lower than expected regret compared with the strategy "treat no patient with surgery." As the highest predicted survival rate based on the model for 2-year survival was $73 \%$, the model would not be able to support the physician decision-making process at any Preg above this value.
Finally, regret-DCA was used to assess decisionmaking when 3-year overall survival was used by the physician as the preferred outcome (Fig 3, C). When considering 3-year survival, for a Preg $<50 \%$, the least regretful strategy was to treat all patients with an operation. In contrast, for values $>50 \%$, the treatment decision based on the model resulted in lower regret. As such, for a Preg $<50 \%$, the least regretful strategy would be to offer liver resection to patients with a predictive 3-year survival $>50 \%$. In contrast, if the Preg was $>50 \%$, the preferred least-regretful therapeutic strategy would be "use a predictive model to select patients who would benefit from surgery." For physicians considering 3-year survival, the highest predicted survival of the model was $62 \%$, and therefore, the model would not be able to support the physician in the decision process above this threshold.

Survey results. To further assess how physicians make treatment decisions regarding patients with ICC, a survey was conducted among 50 physicians who treat patients with ICC. The survey specifically sought to assess the range of physician Pregs associated with 3 different cases characterized by different predicted survival probabilities (Table III). While all 50 physicians were able to express a value for the regret of omission and commission, not every physician believed they could define an expected survival for the various scenarios. As such, there were 110 answers (40 for case 1, 33 for case 2, and 37 for case 3) defining the expected survival for the patients in scenarios 1,2 , and 3. In looking at all 3 scenarios, resection would have been recommended in $82(74.5 \%)$ cases, whereas no resection and chemotherapy alone would have been recommended in 28 $(25.5 \%)$ cases.

For case 1, the hypothetical patient had a total of 196 points, which resulted in a 1-, 2-, and 3-year model-predicted survival of $70 \%, 45 \%$, and $30 \%$, respectively (Table IV). When actual providers considered case 1 , the reported expected median patient survival was 2 years (IQR, 1-2), which was comparable to the predicted survival of $45 \%$ at 2 years. Physician respondents reported a median Preg of 26\% (IQR, 12-40). Based on these responses, both "treat all patients with surgery" and "use a predictive model to select patients to treat" would result in low expected regret (Fig 3, $B)$. Specifically, with an expected survival of 2 years and a low Preg of 26\%, the regret-DCA suggested that liver resection should be offered to the patient in case 1 . Basing the therapeutic decision on the regret model, $85 \%$ of physicians (34 out 
Table III. Results from the survey on the operative treatment of 3 case scenarios of patients with intrahepatic cholangiocarcinoma (ICC). A total of 50 physicians with expertise in the treatment of ICC replied to the survey, reporting for each patient the supposed expected survival after the operation and their regret of commission and omission on a scale ranging from 0 to 100

\begin{tabular}{lc}
\hline & Median, (IQR) \\
\hline Case 1 & $90(80-100)$ \\
Regret of omission (\%) & $18(10-40)$ \\
Regret of commission (\%) & $26(12-40)$ \\
Threshold probability (\%) & $2(1-2)$ \\
Expected survival (y) & $100(100-100)$ \\
Case 2 & $1(1-20)$ \\
Regret of omission (\%) & $5(4-17)$ \\
Regret of commission $(\%)$ & $3(2-3)$ \\
Threshold probability $(\%)$ & \\
Expected survival (y) & $45(12.5-50)$ \\
Case 3 & $50(20-80)$ \\
Regret of omission (\%) & $54(30-83)$ \\
Regret of commission $(\%)$ & $1(1-2)$ \\
Threshold probability $(\%)$ & \\
Expected survival (y) &
\end{tabular}

$I Q R$, Interquartile range.

of 40) would have decided for the same treatment.

For case 2, the median Preg was 5\% (IQR, 417). The median reported expected survival was 3 years (IQR, 2-3), which was comparable to the predicted survival of $55 \%$ at 3 years. The use of the regret-DCA would have led all the physicians (33 out of 33) to decide for hepatic resection.

Regarding case 3, the median Preg was 54\% (IQR, 30-83). The median reported expected survival was 1 year (IQR, 1-2), which was comparable to the predicted survival of $52 \%$ at 1 year, possibly leading all physicians to decide to prefer chemotherapy to an operation. Using the prediction model, the patient in case 3 had a total of 319 points, yielding a predicted 1-, 2-, and 3-year survival of $52 \%, 30 \%$, and $15 \%$, respectively. With an expected survival of 1 year and a Preg of $54 \%$, the regret-DCA would suggest that most physicians not offer liver resection to this patient. Basing the therapeutic decision on the regret model, $60 \%$ of physicians (22 out of 37) would have decided for the same treatment.

\section{DISCUSSION}

In the medical setting, classic decision-making theory defines rationality as aiming to maximize the outcome that follows a decision to perform a
Table IV. Survival predictions based on our nomogram for the 3 cases presented in the survey

\begin{tabular}{|c|c|}
\hline & Points \\
\hline \multicolumn{2}{|l|}{ Case 1} \\
\hline \multicolumn{2}{|l|}{ Patients Characteristics } \\
\hline Tumor Size: 5 cm & 96 \\
\hline Node Status: 1 suspicious for metastasis & 100 \\
\hline Vascular Invasion: No & 0 \\
\hline Number of Tumors: Singular & 0 \\
\hline Total Points & 196 \\
\hline \multicolumn{2}{|l|}{ Model Predicted Survival } \\
\hline $1 \mathrm{y}$ & $70 \%$ \\
\hline $2 y$ & $45 \%$ \\
\hline $3 y$ & $30 \%$ \\
\hline \multicolumn{2}{|l|}{ Case 2} \\
\hline \multicolumn{2}{|l|}{ Patients Characteristics } \\
\hline Tumor Size: 3 cm & 0 \\
\hline Node Status: 0 suspicious for metastasis & 0 \\
\hline Vascular Invasion: No & 0 \\
\hline Number of Tumors: Singular & 0 \\
\hline Total Points & 0 \\
\hline \multicolumn{2}{|l|}{ Model Predicted Survival } \\
\hline $1 \mathrm{y}$ & $85 \%$ \\
\hline $2 y$ & $70 \%$ \\
\hline $3 \mathrm{y}$ & $55 \%$ \\
\hline \multicolumn{2}{|l|}{ Case 3} \\
\hline \multicolumn{2}{|l|}{ Patients Characteristics } \\
\hline Tumor Size: $>5$ cm & 96 \\
\hline Node Status: 1 suspicious for metastasis & 100 \\
\hline Vascular Invasion: Yes & 80 \\
\hline Number of Tumors: 3 Nodules & 43 \\
\hline Total Points & 319 \\
\hline \multicolumn{2}{|l|}{ Model Predicted Survival } \\
\hline $1 \mathrm{y}$ & $52 \%$ \\
\hline $2 y$ & $28 \%$ \\
\hline $3 \mathrm{y}$ & $12 \%$ \\
\hline
\end{tabular}

specific treatment. ${ }^{21}$ However, this approach to decision-making analysis is likely inadequate in those clinical scenarios associated with high levels of uncertainty, such as the decision to perform an operation on a patient with ICC. While an operation benefits many patients with ICC, previous large multi-institutional studies on patients undergoing liver resection for ICC have described mixed cohorts of patients, some of whom benefitted from an operation and others with a prognosis comparable to patients who did not have an operation. ${ }^{6,7}$

Furthermore, guidelines to assist physicians in the preoperative selection of patients with ICC who might benefit the most from liver resection are lacking. ${ }^{22,23}$ As such, models considering the effects that anticipatory regret may have on preoperative decision-making can lead to more wellconsidered choices. ${ }^{24}$ Bell et $\mathrm{al}^{25}$ describe regret 
as "a psychological reaction to making a wrong decision, when wrong is determined on the basis of actual outcomes rather than on the information available at the time of the decision." Regret model theory has been applied to analyze the decisionmaking for the operative treatment of other hepatopancreaticobiliary malignancies such as hepatocellular carcinoma and pancreatic ductal adenocarcinoma. ${ }^{13,15,26}$

Specifically, as noted in the paper by Cucchetti et al, ${ }^{13}$ regret theory provides a new perspective for treatment-related decisions applicable to the clinical setting. The theory explicitly brings to light the otherwise implicit "biases" or "perspectives" of different physicians when they are considering whether to offer therapy to a patient. The theory helps quantitate the variability in whether the same patient is offered a specific therapy based on the different amounts of decisional regret the provider is willing to tolerate.

In the current paper, we sought to apply regretbased DCA to the decision-making process in the setting of the operative treatment of ICC patients. While integrating medical knowledge with "physician attitudes" in decision-making occurs during the everyday practice of performing operations, this process is implicit and "qualitative." The importance of our study is that our model specifically quantifies various elements of decisionmaking. In turn, we were able to define how decisions rely on medical knowledge (postoperative survival predicted by a nomogram, severity disease assessment) and physician attitudes (regret of commission and omission). Specifically, we quantitatively examined the changes in surgeon regret that can lead to different recommendations.

In essence, we made more explicit the implicit variation in decisional regret that frequently influences a surgeon's willingness to provide an operation. To this end, we developed and evaluated the performance of a preoperative model to support physicians in selecting patients who might benefit the most from hepatectomy. More importantly, using regret-based DCA, our approach incorporated a decision maker's preferences from the perspective of regret by estimating a threshold probability for a decision maker. To our knowledge, this is the first application of regret DCA to assist surgeons in decision-making for patients with ICC tumors. The decision regarding resection for patients with ICC is particularly well suited for a regret-based approach, given the general poor prognosis of this disease leading to the need for personalized patient care.
Two different nomograms have previously been proposed to predict the prognosis of patients with ICC following operative resection. ${ }^{6,27}$ In one study, Wang et $\mathrm{al}^{27}$ proposed a nomogram that included serum carcinoembryonic antigen $(\mathrm{HR}=1.00)$, CA 19-9 $(\mathrm{HR}=1.01)$, tumor diameter $(\mathrm{HR}=1.07)$ and number (2-3 nodules, HR $=1.58$; $>3, \mathrm{HR}=6.09$ ), vascular invasion $(\mathrm{HR}=1.60)$, and lymph node metastasis $(\mathrm{HR}=2.05)$ as well as direct invasion/ local extrahepatic metastasis $(\mathrm{HR}=1.59)$. The authors reported that this model performed well on internal validation with a $\mathrm{C}$-index of 0.74 .

In a separate study, Hyder et $\mathrm{al}^{6}$ proposed a nomogram that included factors such as age at diagnosis $(\mathrm{HR}=1.31)$, tumor size $(\mathrm{HR}=1.50)$, multiple tumors $(\mathrm{HR}=1.58)$, cirrhosis $(\mathrm{HR}=1.51)$, lymph node metastasis $(\mathrm{N} 1$, $\mathrm{HR}=1.78 ; \mathrm{Nx}, \mathrm{HR}=1.29)$, and vascular invasion (microscopic, HR = 0.94; macroscopic, HR $=2.10)$. This proposed nomogram demonstrated good predictive abilities with a C-index of 0.69 . These nomograms were, however, designed to be used in the postoperative setting and included information largely available only on final operative pathologic assessment. As such, neither nomogram is applicable to the preoperative setting.

In the current study, we sought to develop a nomogram that could be used in the preoperative setting to help direct patient treatment. Factors including tumor size, number of tumors, major vascular invasion, and the presence of suspicious lymph nodes on cross-sectional imaging were included in the preoperative nomogram based on multivariable analysis. Internal validation of the model with a bootstrapping resample method $(n=5,000)$ demonstrated good accuracy (C-index $=0.59)$, although-as expected-lower accuracy than the postoperative nomograms, which incorporate more details from the pathologic specimen. The graphs comparing predicted and observed survivals at 1,2 , and 3 years did demonstrate good calibration of the nomogram (Supplementary Fig, $A, B$, and $C$ ), suggesting that such a model may be a helpful tool to assist physicians in estimating prognosis in the preoperative setting. Importantly, the nomogram also demonstrated significant heterogeneity in the prognosis of patients with ICC (eg, case 1 versus case 2 versus case 3), suggesting that the benefits of operative resection were highly varied among patients.

While use of nomograms in the preoperative period may be helpful, other factors in the clinical setting may also be important to facilitate decisionmaking. In particular, regret theory postulates that 
choices may be influenced by the decision maker's anticipation that certain outcomes will be associated with high regret, which the provider would like to minimize or avoid. ${ }^{12,13,15-17}$ Regret-based DCA is particularly relevant for decisions characterized by uncertainty and consequences that are impossible to predict at the time of the decision. After making a decision in the setting of uncertainty, the physician may discover that an alternative approach would have been preferable when he or she learns the outcomes, bringing about a sense of loss or regret. If we hope to minimize regret, the "best" choice would be the one associated with the least amount of regret. Importantly, the assessment of regret of commission versus omission can be used to compute a threshold value at which the physician is uncertain about which treatment strategy to adopt. ${ }^{11,12}$

Our data suggest that regret theory can be applied to decision-making for operation versus avoiding an operation for patients with ICC. Given the heterogeneous nature of ICC patients, one single treatment recommendation may not be appropriate for all patients. In such a setting, the physician's preference will likely play an important role in the treatment recommendation. Data from the current study demonstrate that the "best" therapeutic option may include either liver resection or no liver resection/chemotherapy only, depending on the provider's preferences expressed by the regret threshold values. Our data explicitly demonstrate how either liver resection or no liver resection/chemotherapy alone may be recommended based on the feeling of the provider who is participating in the decision. By accounting for personal regret about liver resection compared with the alternative treatment of chemotherapy alone, providers can arrive at the final decision based on a combination of the expected outcome as well as the least amount of regret. ${ }^{13}$ In this manner, the decision-making process includes both feelings of intuition as well as logical analysis and deliberation. ${ }^{13}$ In turn, physicians are forced more fully to evaluate their personal feelings about the decision-making process.

In addition to modeling regret-based DCA, we also performed an assessment of regret and decision-making based on a 50-physician survey. As part of the survey, we asked the respondents to express their expected regret regarding the treatment decisions around 3 different clinical scenarios. The survey corroborated the utility of the regret-DCA. Interestingly, the Pregs varied across a wide range of values, emphasizing how different physicians can suggest different therapies. Still, the application of the regret DCA demonstrated that most physicians act according to the regretthreshold model, leading to similar decisions regarding which treatment to implement, even when there is high uncertainty. ${ }^{13}$

For example, when combining the results of the survey with the regret-DCA, we were able to appreciate a good correlation between the severity of the clinical scenario and the decision to treat the patient. In case 3 , for instance, the patient had an advanced ICC (3 lesions, the major measuring $>5 \mathrm{~cm}$, one suspicious positive lymph node, and vascular invasion) with a survey-based expected survival of 1 year and a threshold probability of $54 \%$ (IQR, 30-83). The regret-DCA analysis demonstrated that even if the patient presented with a technically resectable ICC, more than half of physicians would withhold an operation because of the expected regret associated with the dismal postoperative prognosis.

Conversely, case 2 presented an early ICC (single nodule measuring $3 \mathrm{~cm}$, no suspicious positive lymph nodes or vascular invasion at imaging). Not surprisingly, with an expected survival of 3 years, physicians reported a regret of omission of $100 \%$ (IQR, 100-100) and a regret of commission of only $1 \%$ (IQR, 1-20), resulting in a threshold probability of 5\% (IQR, 4-17). The regret-DCA analysis demonstrated that all the physicians who replied to the survey would suggest an operation for such a patient with an early ICC. As such, these survey results demonstrate how the combination of rational strategies, such as a prognostic nomogram, can be incorporated with regret-DCA to select patients with ICC for operative treatment.

The current study has several limitations. First, as noted, the proposed preoperative nomogram had a lower prediction ability compared with the previously reported postoperative nomograms. 6,27 While this was expected, given the inclusion of only preoperative factors, further studies that focus on improving the proposed preoperative model are needed. Other limitations pertain to the decision-making analysis itself. Operative regret may also be dependent on a surgeon's experience, age, and his or her own outcomes. These factors were not explicitly included or analyzed in our model but were implicitly part of the surgeon survey.

In addition, while we considered the decision to offer ICC at a single time point, many of these decisions happen over a period of time and may involve different levels of regret at different time points. 
Moreover, the model presumed a single decision maker who incorporated individual's level of regret. However, in reality, decisions-including those around level of regret-involve a multidisciplinary team and the patient. As such, the approach to understanding decision-making needs to be multifaceted. Our first goal was to define the amount of decisional regret at the provider level and understand how this might affect decisions. These data are important to patients because they demonstrate that decisions regarding their care are not always being driven by "objective" diseasebased factors. Rather, physician perceptions/attitudes often impact provider recommendations. These data are important to patients as they allow the patient to better understand this dynamic and discuss with the provider how the element of regret, error of omission versus commission, might be at play in the decisions being discussed. The second part of the current project, which is underway, is prospectively evaluating patient regret following an operation.

In conclusion, the regret-based DCA may provide a more appropriate framework for physicians in the decision-making process to recommend an operation for ICC patients. Currently, a model that integrates a predictive tool (staging system or nomogram) and treatment decision is not available for the management of ICC. The current study provides an example of a regret-based DCA that includes a simple and clinically useful nomogram, while also incorporating more intuitive factors of decision-making, such as regret. Tools that incorporate both rational and intuitive elements are likely to be helpful in supporting physicians in their decision to offer liver resection to patients with ICC.

\section{SUPPLEMENTARY DATA}

Supplementary data related to this article can be found online at http://dx.doi.org/10.1016/j.surg.2016.01.023.

\section{REFERENCES}

1. Cardinale V, Semeraro R, Torrice A, Gatto M, Napoli C, Bragazzi MC, et al. Intra-hepatic and extra-hepatic cholangiocarcinoma: new insight into epidemiology and risk factors. World J Gastrointest Oncol 2010;2:407-16.

2. Lafaro KJ, Cosgrove D, Geschwind JF, Kamel I, Herman JM, Pawlik TM. Multidisciplinary care of patients with intrahepatic cholangiocarcinoma: updates in management. Gastroenterol Res Pract 2015. [Epub ahead of print].

3. Bridgewater J, Galle PR, Khan SA, Llovet JM, Park JW, Patel T, et al. Guidelines for the diagnosis and management of intrahepatic cholangiocarcinoma. J Hepatol 2014;60: 1268-89.
4. Nathan H, Aloia TA, Vauthey JN, Abdalla EK, Zhu AX, Schulick RD, et al. A proposed staging system for intrahepatic cholangiocarcinoma. Ann Surg Oncol 2009;16: 14-22.

5. de Jong MC, Nathan H, Sotiropoulos GC, Paul A, Alexandrescu S, Marques H, et al. Intrahepatic cholangiocarcinoma: an international multi-institutional analysis of prognostic factors and lymph node assessment. J Clini Oncology 2011;29:3140-5.

6. Hyder O, Marques H, Pulitano C, Marsh JW, Alexandrescu S, Bauer TW, et al. A nomogram to predict long-term survival after resection for intrahepatic cholangiocarcinoma: an Eastern and Western experience. JAMA Surg 2014; 149:432-8.

7. Valle J, Wasan H, Palmer DH, Cunningham D, Anthoney A, Maraveyas A, et al. Cisplatin plus gemcitabine versus gemcitabine for biliary tract cancer. N Engl J Med 2010;362:1273-81.

8. Spolverato G, Yakoob MY, Kim Y, Alexandrescu S, Marques HP, Lamelas J, et al. Impact of complications on long-term survival after resection of intrahepatic cholangiocarcinoma. Cancer 2015;121:2730-9.

9. Spolverato G, Maqsood H, Vitale A, Alexandrescu S, Marques HP, Aldrighetti L, et al. Readmission after liver resection for intrahepatic cholangiocarcinoma: a multiinstitutional analysis. J Gastrointest Surg 2015;19:1334-41.

10. Cillo U, Spolverato G, Vitale A, Ejaz A, Lonardi S, Cosgrove D, et al. Liver resection for advanced intrahepatic cholangiocarcinoma: a cost-utility analysis. World J Surg 2015;39:2500-9.

11. Tsalatsanis A, Hozo I, Vickers A, Djulbegovic B. A regret theory approach to decision curve analysis: a novel method for eliciting decision makers' preferences and decision-making. BMC Med Inform Decis Mak 2010;10:51.

12. Tsalatsanis A, Barnes LE, Hozo I, Djulbegovic B. Extensions to regret-based decision curve analysis: an application to hospice referral for terminal patients. BMC Med Inform Decis Mak 2011;11:77.

13. Cucchetti A, Djulbegovic B, Tsalatsanis A, Vitale A, Hozo I, Piscaglia F, et al. When to perform hepatic resection for intermediate-stage hepatocellular carcinoma. Hepatology 2015;61:905-14.

14. Hernandez JM, Tsalatsanis A, Humphries LA, Miladinovic B, Djulbegovic B, Velanovich V. Defining optimum treatment of patients with pancreatic adenocarcinoma using regret-based decision curve analysis. Ann Surg 2014;259:1208-14.

15. Djulbegovic B, Hozo I, Schwartz A, McMasters KM. Acceptable regret in medical decision making. Med Hypotheses $1999 ; 53: 253-9$.

16. Smith RD. Is regret theory an alternative basis for estimating the value of healthcare interventions? Health Policy 1996;37:105-15.

17. Loomes G, Sugden R. Regret theory: an alternative theory of rational choice under uncertainty. Econ J 1982;92:805-24.

18. R Core Team. R: a language and environment for statistical computing. Vienna: R Foundation for Statistical Computing; 2014.

19. Harrell FE. Hmisc: Harrell miscellaneous. Nashville: Frank Harrell; 2015.

20. Therneau T. A package for survival analysis in S; 2015. Available from: http:/ /CRAN.R-project.org/package=survival.

21. Hunnik M, Glasziou P. Decision-making in health and medicine. Integrating evidence and values. Cambridge: Cambridge University Press; 2001. 
22. Spolverato G, Vitale A, Cucchetti A, Popescu I, Marques HP, Aldrighetti L, et al. Can hepatic resection provide a longterm cure for patients with intrahepatic cholangiocarcinoma? Cancer 2015;121:3998-4006.

23. Weber SM, Ribero D, O'Reilly EM, Kokudo N, Miyazaki M, Pawlik TM. Intrahepatic cholangiocarcinoma: expert consensus statement. HPB (Oxford) 2015;17:669-80.

24. Hozo I, Djulbegovic B. When is diagnostic testing inappropriate or irrational? Acceptable regret approach. Med Decis Making 2008;28:540-53.
25. Bell DE, Raiffa H, Tversky A. Decision making: descriptive, normative, and prescriptive interactions. Cambridge (UK): Cambridge University Press; 1988.

26. Hozo I, Schell MJ, Djulbegovic B. Decision-making when data and inferences are not conclusive: risk-benefit and acceptable regret approach. Semin Hematol 2008;45: $150-9$.

27. Wang Y, Li J, Xia Y, Gong R, Wang K, Yan Z, et al. Prognostic nomogram for intrahepatic cholangiocarcinoma after partial hepatectomy. J Clin Oncol 2013;31:1188-95. 